\title{
GENETIC AND EPIGENETIC CONTROL OF SEVERAL ISOZYMIC SYSTEMS IN TETRAHYMENA*
}

\author{
Sally Lyman Allen \\ Department of Zoology, The University of Michigan, \\ Ann Arbor, Mich.
}

One of the important goals of biology today is knowledge of the intracellular organization and its relationship to cell function, not only at one time or under one set of environmental conditions, but also at different times and under changing environmental conditions. One of the ways this relationship has been pursued is by analysis of factors which control the specificity and activity of enzymes. Genes control the specificity of enzymes by determining the linear sequence of amino acids making up component polypeptide chains. They also regulate the formation of these chains either by promoting or preventing transcription, or by their effects on translation. The specific folding of the polypeptides into active enzyme may be conditioned by the presence of small molecules, in the case of allosteric proteins, and, in the absence of these small molecules, conformational changes lead to loss of enzyme activity. Enzymes are characteristically nonrandom in their distribution in the cell. These distributions may be effected by subtle controls over metabolic pools so that a particular enzyme is channeled to a certain pathway, or an enzyme may be membrane bound or delimited so that it is compartmentalized into different cell structures. These seemingly simple distributions have been complicated by the recognition that many enzymes occur in multiple molecular form-or isozymes-and different isozymes of the same enzyme may also be nonrandomly distributed within the cell.

Isozymes add a new dimension to the problem of the relationship between molecular structure and cell function. How do they arise? How are they controlled? Are they fortuitous, representing vestigial molecules left over from evolutionary experimentation? Or, are they functional, endowing cells which possess them with a selective advantage?

Isozymes vary in their molecular relationships and may arise in several ways. They may result from configurational changes in (1) single polypeptide chains (e.g., carbonic anhydrase: Edsall, 1968; penicillinase: Pollock, 1968); (2) polymers of the same chain (e.g., $\beta$-galactosidase: Alpers et al., 1968; glucose-6-phosphate dehydrogenase: Kirkman \& Hanna, 1968); or (3) polymers made up of different polypeptide chains and synthesized either by allelic or nonallelic genes. (For several examples, see review by Shaw, 1965.) The changes in configuration may be brought about by genetic substitution; or,

*Supported by a Research Grant, HD-01243-09, from the National Institute of Child Health and Human Development, U. S. Public Health Service, and by an Institutional Grant, IN-40F, to the University of Michigan from the American Cancer Society. 
by chemical modification, either by (1) differences in the binding of small molecules (e.g., alcohol dehydrogenase: Ursprung, 1968; L-amino acid oxidase: Wellner \& Hayes, 1968); (2) differences in the cleavage of the chain (e.g., possibly, hexokinase: Schimke \& Grossbard, 1968); (3) differences in the degree of polymerization (e.g., $\beta$-galactosidase: Alpers et al., 1968; glucose-6-phosphate dehydrogenase: Kirkman \& Hanna, 1968); or (4) differences in the composition of subunits making up a heteropolymer (e.g., lactate dehydrogenase: Appella \& Markert, 1961; Markert, 1963a). They may also arise without genetic or chemical change, but, as a result of configurational isomerism of the completed molecule (e.g., glutamate dehydrogenase: Sundaram \& Fincham, 1964; malate dehydrogenase (MDH); Kitto et al., 1966). The lactate dehydrogenase (LDH) isozymes are now classic as an example of a well-studied isozymic system. (For a review, see Vesell, 1965.) LDH is a tetramer made up of randomly associated polypeptides synthesized by two nonallelic genes. LDH isozymes show variations which are species dependent, tissue specific, and specific in intracellular location, and they vary in predictable ways with the stage of development and with particular disease states. Chemical differences between LDH isozymes have been observed, such as differences in substrate affinity, allosteric inhibition, and cofactor requirements, and these have been related to functional differences in the isozymes.

Isozymes have been observed for many different enzymes and in organisms as diverse as bacteria, protozoa, maize, Drosophila, and man. This paper will be focussed on the isozymes found in the ciliated protozoan, Tetrahymena pyriformis, syngen 1. Multiple forms of several different enzymes have been observed in Tetrahymena, and variations in the array of forms which are present have been recorded. The kinds of variations which occur here and the nature of their control is the subject of this communication.

\section{Isozymes in Tetrahymena}

Multiple forms of several different enzymes have been observed in crude extracts of $T$. pyriformis by gel electrophoresis. The extracts were prepared in the following manner: The cells were collected by centrifugation at approximately $500 \times g$ for 5 minutes and washed in either glass-distilled water, Dryl's physiological salts solution (Dryl, 1959), or in buffers of various composition. Pellets were measured volumetrically and adjusted to particular concentrations depending upon the enzyme under investigation. Extracts were prepared either by repeated freeze-thawing (6-24 rounds, depending upon the enzyme being studied), by sonication (1-5 $\mathrm{min}$ at $0^{\circ} \mathrm{C}$, using an MSE sonicator set for maximum output), or by extraction in $1-5 \%$ Triton $X-100$. Usually the extracts were used without further treatment, but, in some experiments, additional treatment was carried out. Usually the whole extract was employed, but in a few experiments it was centrifuged at $20,000 \times g$ for ten min, and only the supernatant was used. 


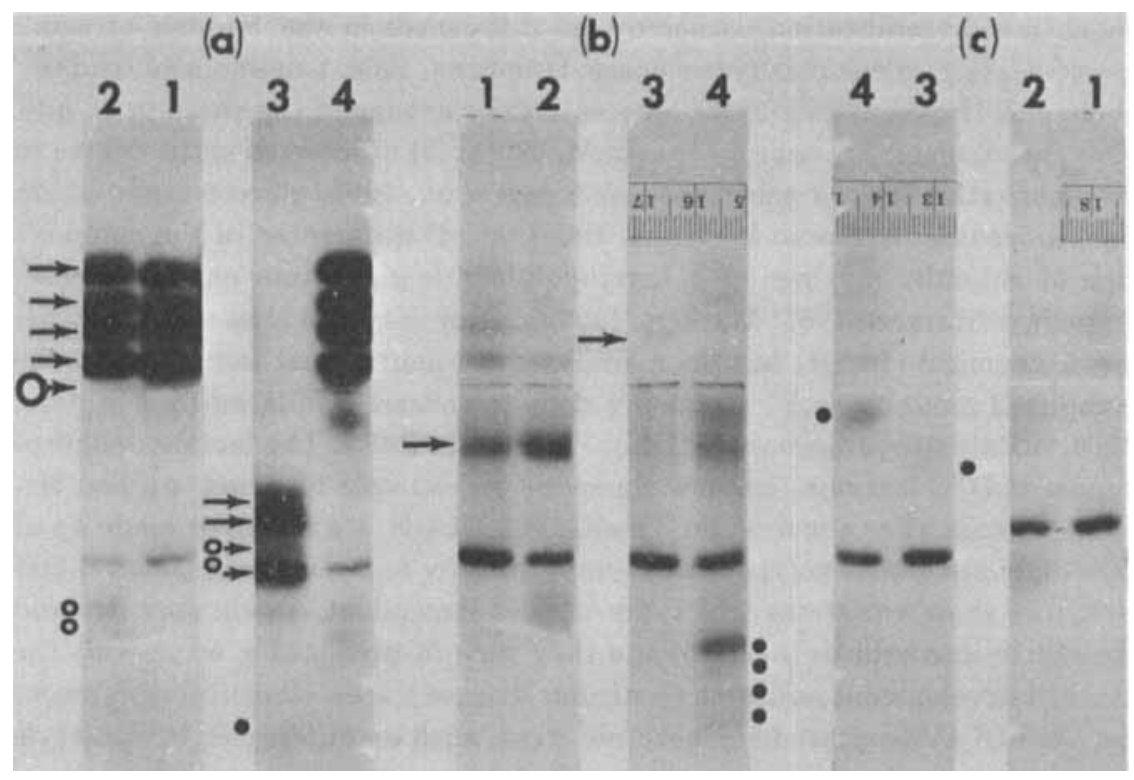

FIGURE 1. Esterases in wild strains of $T$. pyriformis, syngen 1. 1, WH-6; 2, WH-14; 3, UM-226; 4, ALP-4. Two extracts were inserted in each starch gel. The gels were incubated: (a) in $\alpha$-naphthyl propionate in $0.05 \%$ Triton X-100 at $\mathrm{pH} 6.5$; (b) and (c) in $\alpha$-naphthyl butyrate in $0.05 \%$ Triton $\mathrm{X}-100$ at $\mathrm{pH} 6.5$. The whole extract was used in (a) and (b); only the supernatant after centrifugation at $20,000 \times g$ for ten minutes in (c). The esterase- 1 isozymes are indicated by arrows in (a), the esterase- 2 isozymes by arrows in (b). Additional sites of activity in the gels which show strain variation are shown by the open and closed circles. The open circles illustrated by WH-6 and WH-14 correspond to the E-4 isozymes listed in TABLES 1 and 2 in Allen (1965). In this photograph, the cathode is at the top, the anode is at the bottom, and the origin is indicated by 0 .

Multiple forms of the following enzymes have been observed: esterase ( 8 substrates); acid phosphatase ( 6 substrates); dihydronicotinamide-adeninedinucleotide-phosphate $\left(\mathrm{NADPH}_{2}\right)$ oxidase; dihydronicotinamide-adeninedinucleotide $\left(\mathrm{NADH}_{2}\right)$ oxidase; nicotinamide-adenine-dinucleotide-phosphate (NADP) linked isocitrate dehydrogenase (IDH); nicotinamide-adeninedinucleotide (NAD) linked IDH; NADP linked malate dehydrogenase $(\mathrm{MDH})$; NAD linked MDH; glucose-6-phosphate dehydrogenase; $\alpha$-glycerophosphate dehydrogenase; NADP linked glutamate dehydrogenase; choline oxidase; L-leucyl- $\beta$-naphthyl amidase; and $\alpha$-hydroxy acid oxidase. Some of these enzymes have not been extensively investigated or have been difficult to handle (e.g., NAD linked MDH). Illustrative examples of some of the patterns observed are shown in the zymograms appearing in FIGURES 1-3.

The various forms of an enzyme separated by electrophoresis from these crude extracts differ in their molecular relationship. Some are molecules with overlapping specificities, as illustrated by certain of the esterases. These 


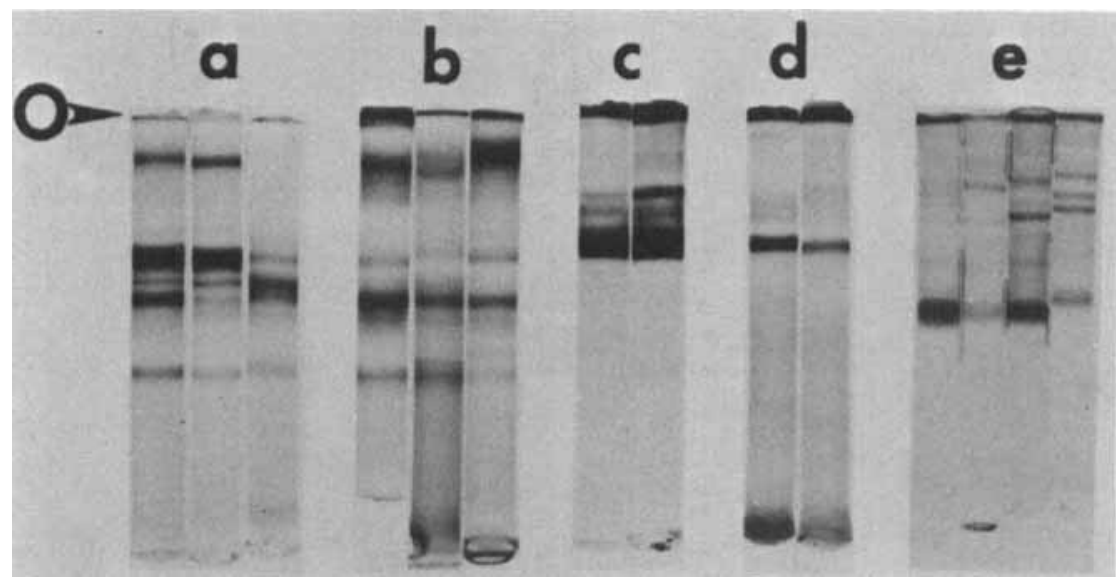

FIGURE 2. (a) $\mathrm{NADPH}_{2}$ oxidase in inbred strains $A 1, \mathrm{C}$, and $\mathrm{D1}$; (b) $\mathrm{NADH}_{2}$ oxidase in inbred strains A1, B and B2; (c) NADP-IDH in inbred strains $\mathrm{Cl}$ and E; (d) NADIDH in inbred strains $A$ and $B$; (e) $\alpha$-hydroxy acid oxidase in inbred strain $B$. The two gels on the right contained extracts which were left on ice for one to two hr before they were frozen-thawed. (a)-(d) were obtained from logarithmic cultures; (e) from stationary cultures. Disc electrophoresis was carried out by the method of Davis (1964). In (e), electrophoresis was stopped after the running dye had migrated two cm, or half the distance used for the gels in (a)-(d). The gels were incubated: in (a) for $15 \mathrm{~min}$ at $25^{\circ} \mathrm{C}$ in $\mathrm{NADPH}_{2}(1 \mathrm{mg} / \mathrm{ml}$ ) and nitroblue tetrazolium (NBT; $1 \mathrm{mg} / \mathrm{ml}$ ) in $0.05 \mathrm{M}$ Tris (hydroxymethyl) aminomethane- $\mathrm{HCl}$ buffer at $\mathrm{pH} 7.5$; in (b) for $60 \mathrm{~min}$ in a reaction mixture similar to (a) except for the substitution of $\mathrm{NADH}_{2}$; in (c) for three min at $25^{\circ} \mathrm{C}$ in 0.025 M D,L-isocitric acid, NADP (1 mg/ml), $\mathrm{MgCl}_{2}(20 \mathrm{mM}), \mathrm{NBT}(1 \mathrm{mg} / \mathrm{ml})$, phenazine methosulfate (PMS; $0.3 \mathrm{mg} / \mathrm{ml}$ ) in $0.05 \mathrm{M}$ glycylglycine buffer at $\mathrm{pH} 6.5$; in (d) for 60 min in a reaction mixture similar to (c) except for the substitution of NAD; in (e) for 30 $\min$ at $25^{\circ} \mathrm{C}$ in $0.05 \mathrm{M} \mathrm{D,L-} \alpha$-hydroxy valeric acid, NBT $(1 \mathrm{mg} / \mathrm{ml})$, and PMS $(0.3 \mathrm{mg} /$ $\mathrm{ml}$ ) in $0.1 \mathrm{M}$ Sörensen's phosphate buffer at $\mathrm{pH} 8$. The cathode is at the top, the anode at the bottom, and the origin is indicated by 0 .

differ from each other in their genetic control, in their biochemical properties, in their response to growth conditions, and in their intracellular distribution. Others are more closely related and have similar biochemical properties. Some are the multiple products of a single gene and may arise by configurational isomerism or by epigenetic change. Some are the products of different alleles of the same gene, and others are the products of intragenic interaction and appear in heterozygotes. In the case of the oxidases and dehydrogenases, too little is known about the properties of the separated enzymatic activities to determine the degree of their molecular relationship. Judging by the similarity in the zymograms of $\mathrm{NADPH}_{2}$ oxidase and of $\mathrm{NADH}_{2}$ oxidase, however, it would appear that there is considerable overlap in the specificity of some of these resolved activities.

Variations of several different kinds are observed in the zymograms. Variations which are clearly strain dependent have been observed in the esterases and acid phosphatases (FIGURES 1,3), and, for some of these, structural genes 


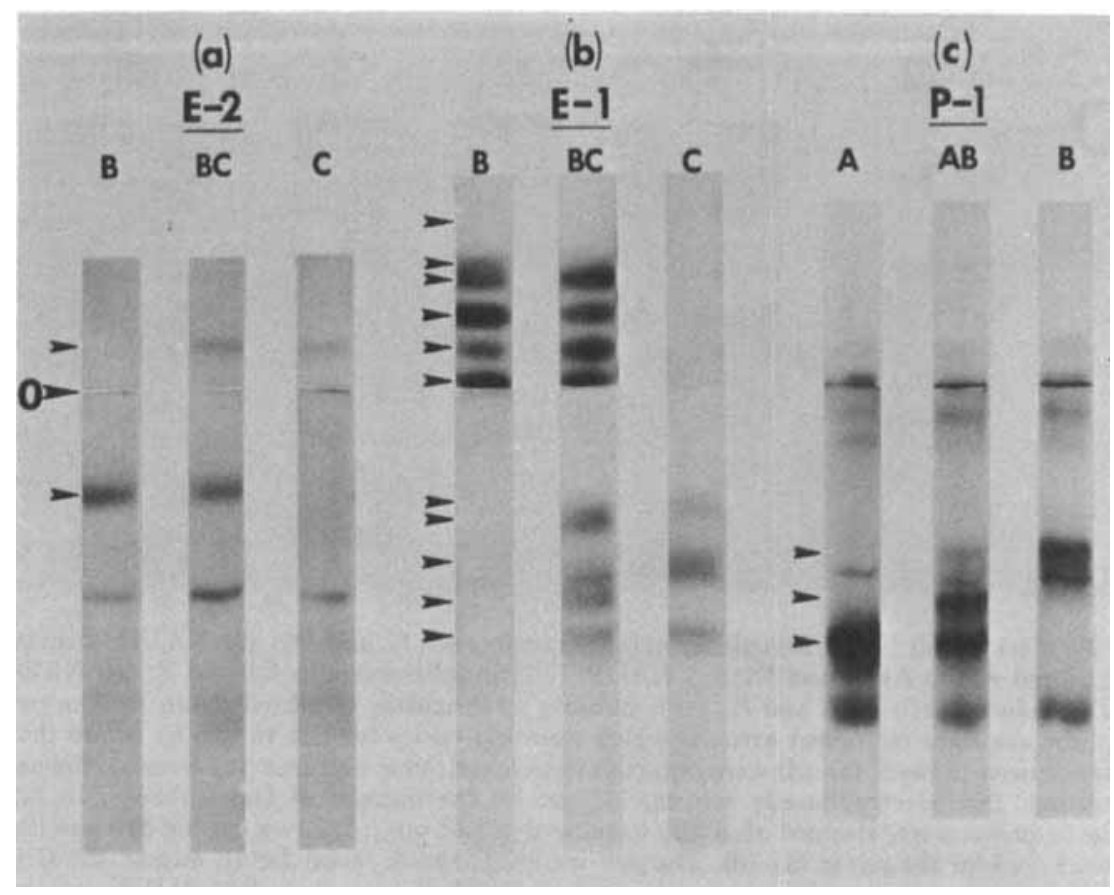

FIGURE 3. Esterases and acid phosphatases in different genotypes of (a) E-2, (b) E-1, and (c) $P$-1. The cathode is at the top, the anode at the bottom, and the origin is indicated by 0 . Specific sites of enzymatic activity are marked by arrows. The starch gels were incubated in (a) $\alpha$-naphthyl butyrate at $\mathrm{pH} 6.5$, (b) $\alpha$-naphthyl propionate at $\mathrm{pH}$ 7.4 , and (c) sodium $\alpha$-naphthyl acid phosphate at $\mathrm{pH} 5$.

have been identified. Another type of variation occurs only in heterozygotes and leads to cells with different stable phenotypes (FIGURES 4,5). Still other variations are unstable, occur in various genotypes, and are dependent upon growth conditions (FIGURES 6,7). This last type of variation has been observed for most of the enzymes examined. Technically, it presents problems, since different cultures do vary in growth rate. Without running samples of cultures grown for varying periods of time, one could easily be misled into mistaking a growth-cycle variation for a strain variation.

\section{Genetically Controlled Variations}

Variations that are clearly genetic have been observed for the esterases and acid phosphatases. Structural genes have been identified for esterase-1, esterase-2, and phosphatase-1 (Allen, 1961; Allen et al., 1963a). Some of the chemical properties of these enzymes were defined in crude extracts and have been discussed at length elsewhere (Allen, 1960, 1965; Allen et al. 1963a, b; 1965). Certain salient features will be summarized here. Esterase-1 is controlled by codominant alleles at the $E$ - 1 locus (FIGURE 3 , column b), and each 


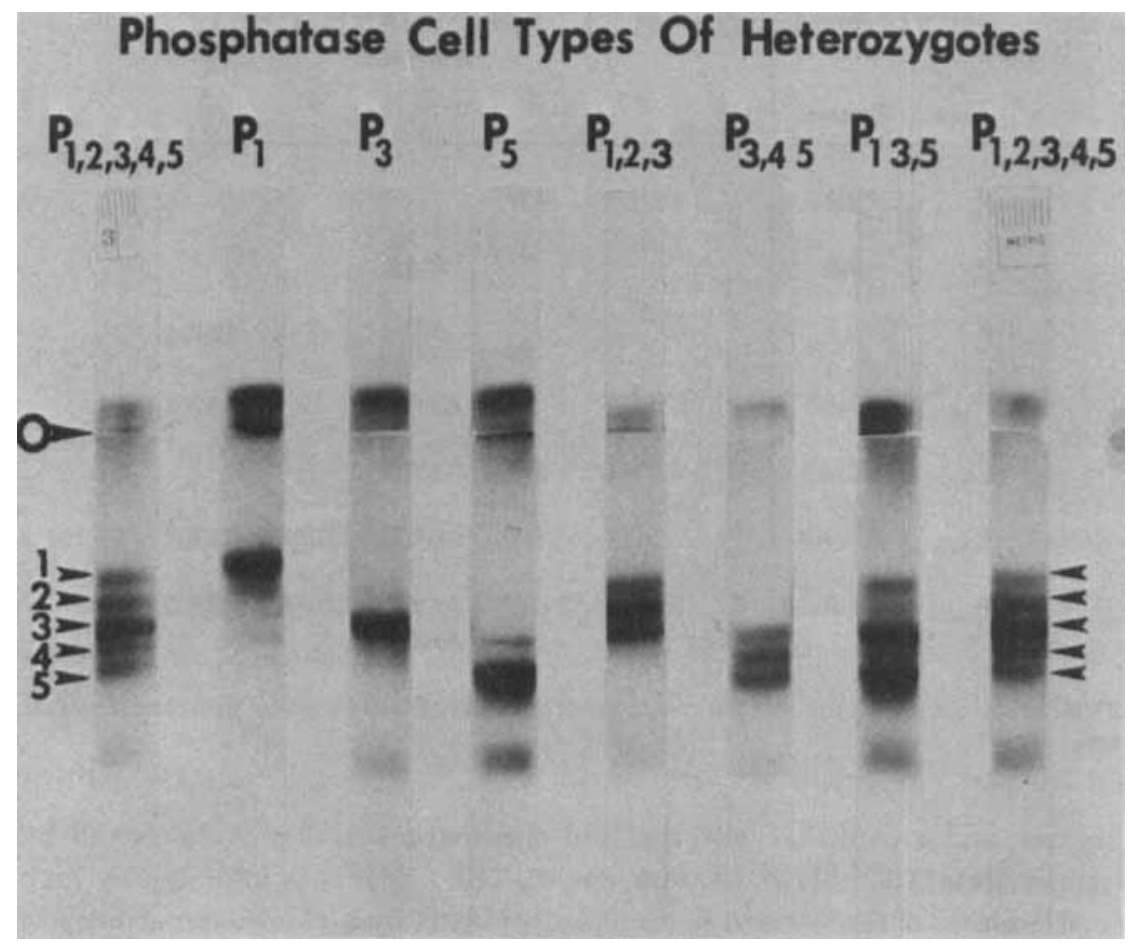

FIGURE 4. Phosphatase- 1 in seven heterozygous cell types. The isozymes are indicated by number. The starch gels were incubated in sodium $\alpha$-naphthyl acid phosphate for one hr at $23^{\circ} \mathrm{C}$ at $\mathrm{pH} 5$. The cathode is at the top, the anode at the bottom, and the origin is indicated by 0 .

homozygote has a group of six or more isozymes. The heterozygote has both groups of isozymes, and no new isozymes have, as yet, been observed in extracts obtained from heterozygotes. The esterase- 1 isozymes are relatively stable; resist treatment with high concentrations of urea, split $\alpha$-naphthyl acetate, and $\alpha$-naphthyl propionate; are activated by sodium taurocholate $\left(10^{-2} \mathrm{M}\right)$, sodium cholate $\left(10^{-2} \mathrm{M}\right)$, and Triton X-100 (FIGURE 1$)$; and are inhibited by eserine sulfate $\left(10^{-4} \mathrm{M}\right)$, L-cysteine $\left(10^{-2} \mathrm{M}\right)$, and mercaptoethanol $\left(10^{-3} \mathrm{M}\right)$. New isozymes which migrate closer to the cathode can be generated from the isozymes of either homozygote if the extracts are treated with iodoacetamide. Conversion of the native isozymes into these new forms is gradual and depends upon the concentration of iodoacetamide and the length of time the extract is treated.

Allelic forms of esterase-2 have been found. Each $E-2$ homozygote has a distinct isozyme (FIGURE 3, column a) and the heterozygote has both isozymes. The esterase- 2 isozymes are less stable than the esterase- 1 isozymes; are inactivated by urea $(4 \mathrm{M})$ and iodoacetamide ( $\mathrm{M})$, split $\alpha$-naphthyl 


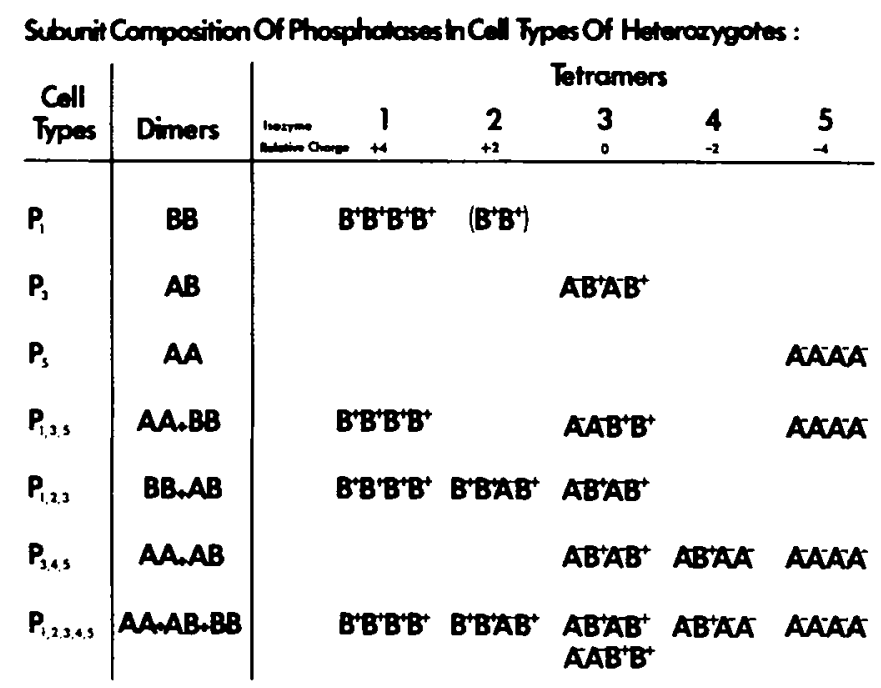

FIGURE 5. Proposed subunit composition of phosphatase-1 in seven heterozygous cell types.

butyrate, and $\alpha$-naphthyl valerate; and enzymatic activity is unaffected by eserine sulfate $\left(10^{-2} \mathrm{M}\right)$ and sodium cholate $\left(10^{-2} \mathrm{M}\right)$. It is inhibited by high concentrations of taurine and sodium taurocholate, by $p$-chloromercuribenzoic acid $\left(10^{-3} \mathrm{M}\right)$, and to some extent by Triton X-100 (FIGURE 1; see also FIGURE 1 in Allen et al., 1965).

Phosphatase- 1 is controlled by codominant alleles at the P-1 locus, and each homozygote has one principal isozyme (FIGURE 3, column c). In the heterozygote, intragenic interaction occurs, and as many as five isozymes have been observed. The phosphatase-1 isozymes split sodium $\alpha$-naphthyl acid phosphate, naphthol AS-phosphate, naphthyl AS-MX phosphate, naphthyl AS-B1 phosphate, sodium $\beta$-glycerophosphate, and glucose-6-phosphate, with a pH optimum of 5. Enzymatic activity is inhibited by sodium fluoride $\left(10^{-2}\right.$ $\mathrm{M})$ and $d$-tartaric acid $\left(10^{-2} \mathrm{M}\right)$, but is unaffected by $p$-chloromercuribenzoic acid $\left(10^{-4} \mathrm{M}\right)$. These isozymes are less stable than the esterase- 1 or esterase-2 isozymes. They are inactivated in $30 \mathrm{~min}$ by temperatures between $40^{\circ} \mathrm{C}$ and $45^{\circ} \mathrm{C}$, by low $\mathrm{pH}(3.8)$ and by slightly alkaline $\mathrm{pH}$ 's $(7-8)$, and by urea in concentrations between 2 and $4 \mathrm{M}$. There are differences in the stability of the five phosphatase-1 isozymes, with Isozyme 1 being less stable than Isozyme 5. Mixed extracts of Isozyme 1 and Isozyme 5, and also an extract containing pure Isozyme 3 , have been subjected to various treatments known to effect dissociation and reaggregation. Freezing in $0.1-2 \mathrm{M} \mathrm{NaCl}$ in buffers of various composition was without effect. In other experiments, acid dissociation was carried out at $\mathrm{pH} 3.8$, and then the extracts were dialyzed against various buffers at $\mathrm{pH} \mathrm{5-7.} \mathrm{This} \mathrm{treatment} \mathrm{has} \mathrm{also} \mathrm{not} \mathrm{been} \mathrm{very} \mathrm{successful.}$ 


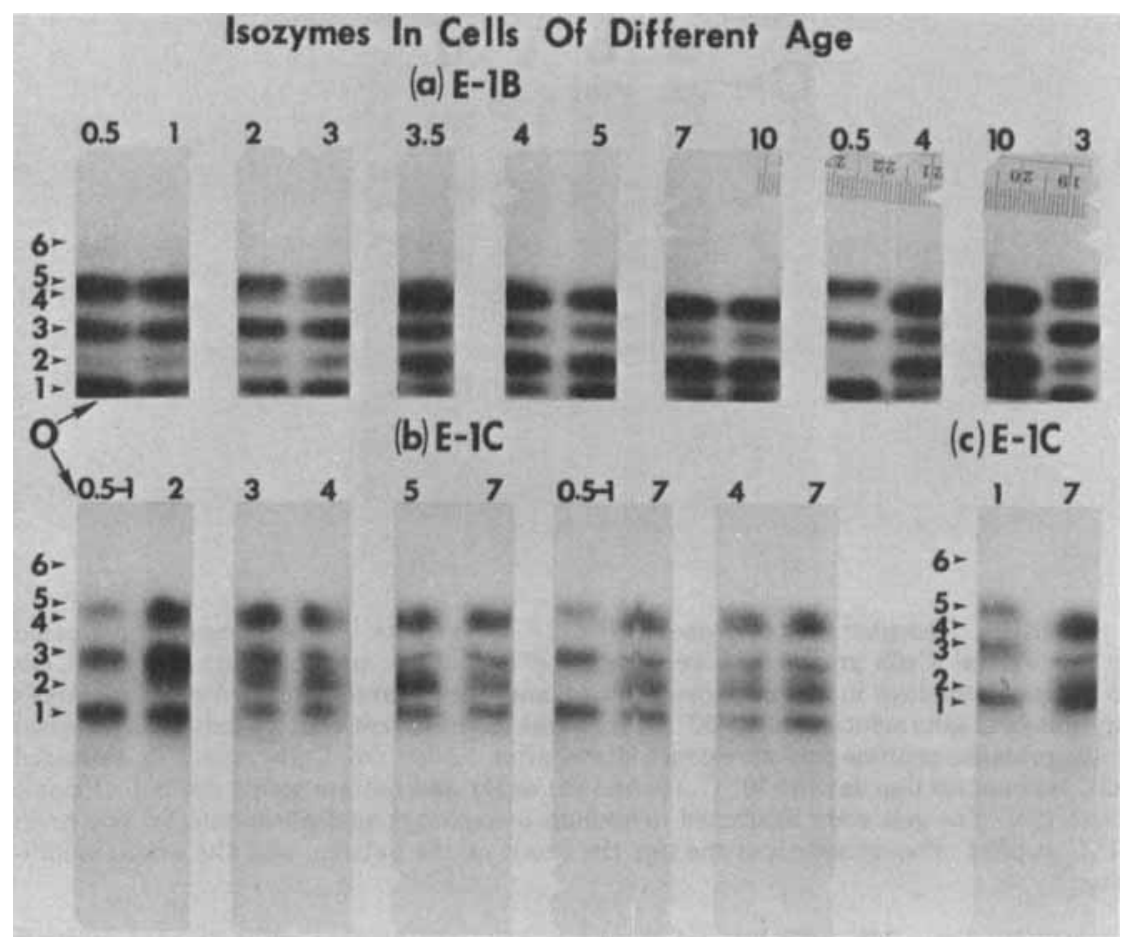

FIGURE 6. Esterase-1 in hompzygotes showing variations during the growth cycle. (a) $E-1^{B} / E-1^{B}$ and (b), (c) $E-1^{C} / E-1^{C}$ cells were grown in $1 \%$ proteose-peptone for the number of days indicated above the starch gels. Each isozyme is identified by a number that appears to the left of the gels. In (b), the bed buffer was pH 7.5; in (c), pH 7.8. Note that C-1 and C-2 are more clearly separated in (b) but that C-4 and C-5 are more clearly separated in (c). The starch gels were incubated in $\alpha$-naphthyl propionate and sodium taurocholate for 1-1.5 hr. The cathode is at the top, the anode at the bottom, and the origin is indicated by 0 .

Very small amounts of other isozymes do appear, but they are almost impossible to resolve by the usual electrophoretic procedures.

Esterase-1, esterase-2, and phosphatase-1 are controlled by genes which appear to be unlinked to each other and to $H$ and $T$, genes specifying two different immobilization antigens (Allen, 1964a; Allen, unpublished; Phillips, 1967a). $E-2$ and $P-1$ are not linked to $m t$, the mating-type locus, but $E-1$ and $m t$ are loosely linked, recombination being of the order of $25 \%$ (Allen, 1964a; Allen, unpublished).

Like most ciliated protozoa, $T$. pyriformis has two types of nuclei, a diploid micronucleus and a polygenomic macronucleus. Tetrahymena has one of each type of nucleus. During conjugation, the macronucleus is normally discarded and replaced by products of the micronucleus. However, in Tetrahymena an abnormal form of conjugation, called genomic exclusion, also occurs, in which the old macronucleus is retained (Allen, 1967a,b; Allen et al., 1967). This 


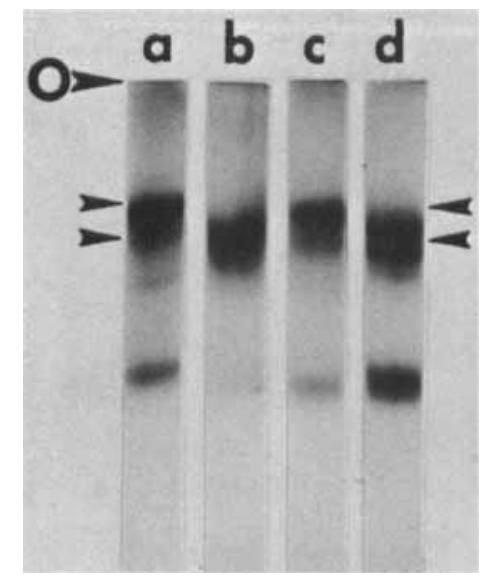

FIGURE 7. Phosphatase-1 isozymes in $P \cdot 1^{B} / P-1^{B}$ extracts. The isozymes are indicated by arrows. (a) Cells grown for five days at $30^{\circ} \mathrm{C}$ in $1 \%$ proteose-peptone; (b) Cells grown for three days in $1 \%$ proteose-peptone and then starved for two days in Dryl's physiological salts solution, all at $30^{\circ} \mathrm{C}$; (c) Same as (b) except that culture was returned to $1 \%$ proteose-peptone and an extract made after $12 \mathrm{hr}$; (d) Cells grown in skimmed milk medium for five days at $30^{\circ} \mathrm{C}$. (a) and (b) or (c) and (d) are strips cut out of single starch gels. The gels were incubated in sodium $\alpha$-naphthyl acid phosphate for one hr at $23^{\circ} \mathrm{C}$ at $\mathrm{pH}$. The cathode is at the top, the anode at the bottom, and the origin is indicated by 0 .

process leads to cells which are heterocaryons and have a micronucleus which can differ in genotype from the old macronucleus. Heterocaryons can be deliberately constructed in which the genes for esterase-1, esterase-2, or for phosphatase-1 differ in the micronucleus and macronucleus. When this was done, it was the form of the enzyme specified by genes in the macronucleus that was expressed. No trace of the enzyme specified by micronuclear genes was found. These observations, like those made earlier in Paramecium aurelia (Sonneborn, 1942), suggest that, when the genes of the macronucleus are active, those in the micronucleus are silent.

\section{Variations in Heterozygotes}

Phenotypic changes have been observed within heterozygous clones of Tetrahymena. These changes are highly stable and lead to cell lines of diverse phenotype. The mechanism by which this change takes place is not understood, although it has been thought not to be due to genetic segregation (Allen, 1965; Nanney, 1964). To be completely objective, its character will not be categorized; hence, the subject will be treated here in a separate section.

The phenomenon of phenotypic drift (also called allelic repression, interallelic interaction, etc.) has been observed not only for heterozygotes at the $E-1, E-2$, and $P-1$ enzyme loci, but also for heterozygotes at the $H$ and $T$ antigen loci (Allen, 1965; Nanney \& Dubert, 1960; Phillips, 1967b). Similar 
observations were also made with the mating-type locus on homozygotes as well as heterozygotes (Allen \& Nanney, 1958). Thus, this phenomenon affects all the identified genes in this organism. For the heterozygotes, the observations can be simply stated: Immediately after the genesis of a heterozygote of hypothetical genotype $A^{1} / A^{2}$, the phenotype associated with both $A^{1}$ and $A^{2}$ is observed within all cells of the clone. When subclones are initiated and propagated for several hundred fissions, it is found that most of these cell lines no longer express both $A^{1}$ and $A^{2}$, but that some lines express the $A^{1}$ phenotype and other lines, the $A^{2}$ phenotype. A few lines still do express both $A^{1}$ and $A^{2}$ but quantitative variations in the relative expression of the alleles are observed in different cells within such lineages. Lines which express $A^{1}$ or $A^{2}$ are completely stable; while those which express both alleles are unstable and give rise to new lines which express only one of the alleles and which are stable. These phenotypic changes appear to be controlled by the macronucleus, since the missing genes reappear during normal conjugation when the old macronucleus is destroyed. The source of these genes is, of course, the micronucleus. During genomic exclusion, the old macronucleus is retained, and, under these conditions, the phenotypic change persists.

Clonal analysis (Allen, 1965; Allen, unpublished observations; Nanney, 1964; Phillips, 1967b) and the analysis of cell pedigrees (Bleyman et al., 1966; Nanney et al., 1964) have shown that some features of phenotypic drift are held in common by all loci but that other features may vary. The properties of phenotypic drift can be summarized by the following:

(1) Phenotypic drift occurs in heterozygotes for all identified (six) loci, but is independent for each locus even when the loci are linked.

(2) The stable cell types that arise have the phenotypes of homozygotes. An exception is a stable phosphatase-1 cell type which is hybrid in phenotype.

(3) Phenotypic drift occurs either if the gene is being expressed, or in the absence of gene expression. In the case of the enzyme loci, the products of both alleles are detected for many fissions $(30-50)$; then one of the products starts to disappear. In the case of the antigen loci, only one of the antigens, $H$ or $T$, is expressed at a particular temperature. If cell lines expressing $H$ are allowed to undergo several fissions and then the temperature is raised, $T$ is expressed. Here, variations are observed in the $T$-phenotype, and they are independent of those observed for $H$.

(4) Phenotypic drift begins at different fissions (f) for different loci: at 0-1 f for $m t$, at $1-2 \mathrm{f}$ for $H$, ten $\mathrm{f}$ for $T, 40 \mathrm{f}$ for $E-1$, and $E-2$, and $50 \mathrm{f}$ for $P-1$.

(5) The probability that allele-1 will be affected rather than allele-2 is equal for those loci in which phenotypic drift begins late $(T, E-1, E-2$, and $P-1)$, and is very skewed for loci in which it occurs early ( $m t$ and $H$ ).

(6) Once phenotypic drift begins, all loci seem to adhere to the same "kinetics"; that is, the frequency of newly formed stable lines is similar (about $1 / 100$ per fission). 
(7) Once a cell line expresses a stable phenotype, no method has been discovered which will effect re-expression of the unexpressed phenotype.

These properties have been used as support for a compound macronucleus made up of diploid subnuclei and as an argument for a particular interpretation of phenotypic drift. This viewpoint has been discussed at length elsewhere (Allen, 1965; Nanney, 1963, 1964). According to this view, the segregation of phenotypes that is observed in heterozygotes does not result because of genetic assortment, but it occurs as a result of factors which affect gene expression in the macronuclear subunit. However, there is no direct experimental evidence for the level at which the genes are affected, and the molecular basis of this phenomenon remains an important, but unsolved, problem.

Even though not understood, the phenomenon of phenotypic drift can be used. It has been particularly useful in providing indirect structural information on the phosphatase-1 isozymes. Clonal analysis of heterozygotes has shown that there are seven cell types, each varying in its synthetic capacity. There is a pattern to these varying synthetic capacities, and this pattern tells us something about the phosphatase molecule and how it is synthesized.

There is a total of five isozymes in a heterozygote. In a newly generated heterozygote, Isozymes 1,3 , and 5 are present. These three isozymes are always found in all cells at this time. Moreover, when cloned, all the cell lines have these three isozymes, and this state of affairs continues for some 50 fissions. Then a change in phenotype occurs, and variations in the isozymic pattern in different cell lines are observed. Some lines have the original three isozymes, but some may have all five isozymes. Other lines have new patterns of three of the isozymes, and still others have only one isozyme.

There are seven qualitatively distinct cell types among the subclones (FIGURE 4). Three cell types have only one isozyme: one has principally Isozyme 1 (the cell type is designated $\left.P_{1}\right)$; one has Isozyme $5\left(P_{5}\right)$; and another has only Isozyme $3\left(P_{3}\right)$. Three cell types have three isozymes: one has Isozymes 1,3 , and 5 , as found in all newly derived heterozygotes $\left(P_{1,3,5}\right)$; one has Isozymes 1,2 , and $3\left(P_{1,2,3}\right)$; and one has Isozymes 3,4 , and $5\left(P_{3,4,5}\right)$. The seventh cell type has all five isozymes $\left(P_{1,2,3,4,5}\right)$.

A clonal analysis was performed on representatives of each of these seven cell types (Allen, 1965). Clones having only one isozyme were found to be completely stable. Thus, all subclones generated from a $P_{3}$ clone, or with Isozyme 3 , had only Isozyme 3 . Similar findings were found for the $P_{1}$ and $P_{5}$ cell types; that is, all subclones derived from a $P_{1}$ clone were $P_{1}$, and all subclones derived from a $P_{5}$ clone were $P_{5}$. Clones typed as $P_{1,2,3,4,5}, P_{1,3,5}, P_{1,2,3}$, and $P_{3,4,5}$ were unstable. Each of these clones gave rise to subclones which varied in phenotype and included new, stable, cell types. Clones having all five isozymes gave rise to all seven cell types; those with three isozymes, to a more restricted array of cell types. Differences were noted in the kinds of stable cell types generated by these clones. All three stable cell types arose from a $\mathbf{P}_{1,2,3,4,5}$ clone, but only two of the three stable types could be derived from 
clones with three isozymes, and each of these gave rise to a different set of types. $P_{1}$ and $P_{5}$ were derived from a $P_{1,3,5}$ clone; $P_{1}$ and $P_{3}$, from a $P_{1,2,3}$ clone; and $P_{3}$ and $P_{5}$, from a $P_{3,4,5}$ clone. Some of these newly derived $P_{1}$, $P_{3}$, and $P_{5}$ subclones were further cloned, and all that were tested were completely stable.

The kinds of stable types that are derived from the different cell types fall into a pattern. This pattern can be interpreted in terms of a hypothetical structure for the phosphatase molecule and can be used to get at how the components that make up the molecule aggregate during the synthesis of this molecule. These ideas are summarized in FIGURE 5.

Since the maximum number of isozymes in the heterozygote is five, the phosphatase molecule is most likely a tetramer. However, if it is a tetramer, complete randomization of the monomers must not occur; otherwise, one would expect only the cell types $P_{1,2,3,4,5}$ and perhaps $P_{1}$ and $P_{5}$ to appear. The data can best be interpreted by assuming that dimers are formed first, and these dimers then associate at random to form the tetramer. The observations suggest that there are three types of dimers, AA, BB, and AB, and that cells differ in their capacity to synthesize particular dimers. Some synthesize only one, others two, and a few all three of them. The stable cell types, $P_{1}, P_{3}$, and $P_{5}$, are those which synthesize only one type of dimer. Apparently, a newly derived heterozygote synthesizes only the dimers $A A$ and $B B$, and either does not synthesize the $A B$ dimer or produces it in undetectable amounts. Once a cell acquires the ability to synthesize $\mathrm{AB}$, the other cell types can be detected. It is interesting that the $P_{3}$-cell type which synthesizes only $\mathrm{AB}$ appears to be as stable as those synthesizing only AA or only $\mathrm{BB}$. This observation is undoubtedly significant, but its meaning is by no means clear.

\section{Epigenetic Variations}

Variations which depend upon the specific conditions of growth of the organism have been observed in all strains and with most of the enzymes that have been examined. These variations are unstable in the sense that they are observed under one set of conditions and not under other conditions. They are perhaps the most elusive to study because of their seeming complexity. The chief factors that elicit variations of this type are (1) the growth cycle, (2) the composition of the medium, and (3) the temperature at which the cultures are grown. The latter has been least studied; but early observations on the esterase-1 isozymes showed a temperature effect (Allen, 1960).

Growth cycle variations have been observed with a number of different enzymes, but the most extensively examined are the esterase- 1 isozymes. Each homozygote has a group of isozymes, and variations in the activity of a given isozyme are observed as a function of the growth cycle. Some isozymes appear sooner than others during logarithmic growth, and some do not reach peak activity until the stationary period (FIGURE 6). Physical and chemical 
treatments of the extracts containing these isozymes showed some difference in the response of individual isozymes (Allen, 1965). In cell fractions obtained by differential centrifugation, the isozymes were nonrandomly distributed among the fractions. The isozyme first to appear during logarithmic growth was enriched in the microsomal fraction (Allen, 1964b).

The most remarkable feature of this study was the observation that isozymes in the two allelic groups showed homologous behavior, and the same relationship between isozymes of a group was retained even though the electrophoretic mobility of the entire group had been shifted. Thus, the responses of homologous isozymes (e.g., Isozyme 3 of E-1b and E-1c) to growth conditions, to chemical treatment, or to centrifugation was similar. With iodoacetamide treatment, new isozymes, more basic in charge, were elaborated from the native isozymes of a group. These observations suggested that the genetic alteration must not occur at the active site of the enzyme nor at a site involved in the configurations necessary for elaborating isozymes, whatever they may be. An hypothesis was developed from the finding that the isozyme first to appear in the growth cycle was also associated with microsomes. It was postulated that this isozyme (Isozyme 3 ) was closest in structure to the newly synthesized form of the enzyme and that the other isozymes were elaborated from this form by associations with other molecules that affected charge and which resulted in attachment to different intracellular structures. The results of treatment of esterase-1 isozymes with iodoacetamide strengthened this view although they provided no insight into mechanism.

Different media, or changing the composition of a medium, result in variations in isozymes. As an illustration of this point, variations in the phosphatase- 1 isozymes will be discussed. First, it should be noted that under conditions of growth which presumably do not favor the formation of lysosomes, phosphatase- 1 may be absent. Certainly it is very greatly reduced in activity. A previous study showed that the type of medium in which the cells were grown resulted in differences in the presence or absence of certain acid phosphatases (Allen et al., 1963b). Phosphatase-1 was present in cells grown in $1 \%$ proteose-peptone or in Cerophyl-Aerobacter medium, but it was not present when cells were grown in synthetic medium. These results were interpreted as one line of evidence in support of a lysosomal location of these acid phosphatases, since lysosomes are presumably elaborated in connection with food vacuoles (Müller and Törö, 1962), and vacuoles are formed in only the first two types of media. Experiments directed at probing this relationship in depth are in progress. Second, some subtle differences have been found in the activities and mobilities of the isozymes found in $P_{1}$ cells. Actually, $P_{1}$ cells have Isozymes 1 and 2 , and even traces of an isozyme with a mobility very similar to Isozyme 3 are found in some extracts. In the standard medium ( $1 \%$ proteose-peptone), the principal isozyme is Isozyme 1 (FIGURE 7, column a). If a culture is grown in peptone for three to five days, then the peptone is removed by centrifugation and the culture is washed and suspended in 
glass-distilled water or in Dryl's solution with sterile technique, and an extract is made after $48 \mathrm{hr}$ of starvation, discrete isozymes are not observed and "muddy" activity is observed (FIGURE 7, column b). When the starved culture is recentrifuged and returned to peptone, the original pattern reappears by $12 \mathrm{hr}$ of growth (FIGURE 7, column c). Another pattern is observed when a culture is grown for five to seven days in a skimmed milk medium (FIGURE 7 , column d). In these extracts, Isozymes 1 and 2 appear equally active, and their mobility has shifted slightly to the anode. These effects, though small, are repeatable, but they are not understood.

\section{Discussion}

Variation in an enzyme takes several forms. An enzyme may be observed to be present or absent; altered in its electrophoretic mobility or some other property, but not in its catalytic properties; altered in its catalytic properties, but not in its electrophoretic mobility; or altered in both; and, finally, isozymes of this enzyme may appear.

Variations of some of these types have been observed in Tetrahymena. An enzyme may be observed to be present or absent. There are two examples of this sort of variation: (1) Phosphatase-1 is almost absent under conditions of growth which prevent lysosomes from forming; this applies to both allelic forms of this enzyme. (2) Variations in the presence or absence of allelic forms are observed in different heterozygous cell lines. This applies to esterase-1, esterase-2, and phosphatase- 1 . In the latter case, a hybrid form of the enzyme may be synthesized to the exclusion of the parental forms of the enzyme. An enzyme may be altered in its electrophoretic mobility, but not in its catalytic properties. Differences in mobility may arise with genic substitution as exemplified by the allelic forms of esterase-1, esterase-2, and phosphatase-1. An enzyme may be altered in its catalytic properties, but not in its electrophoretic mobility; or in both. In Tetrahymena, variations which affect the catalytic properties of an enzyme have not been analyzed. Such variations might be sought by comparing the different members of a family of enzymes, such as the esterases or the phosphatases, which show overlapping and relatively nonspecific activity against the substrates usually employed. Isozymes may appear. They may occur only in certain genotypes, or only in some of the cells of a given genotype, or only under certain conditions of growth.

The molecular bases for the different kinds of isozyme variation observed in Tetrahymena are unknown, although it seems obvious that various levels of control which affect the synthesis or activity of an enzyme are involved. Either genetic or epigenetic control may be exerted over those cases in which presence or absence of isozymes is involved. Epigenetic control is more likely for those cases in which the activity or mobility of an isozyme is affected.

Phosphatase-1 appears to be made up of subunits, as has been found for many other enzymes. (For examples, see Shaw, 1965.) New isozymes appeared in heterozygotes, presumably as a result of the aggregation of dissimilar sub- 
units, in this case, specified by alleles. The total number observed in heterozygotes can be used to estimate the size of the polymer (Shaw, 1964); and, since a total of five isozymes was observed, phosphatase-1 is most likely a tetramer. Not all of the isozymes were found in all of the cells. The patterns observed in different clones suggested that there are constraints in the association of the component subunits and that different subunits are differentially synthesized or utilized. Observations somewhat similar to these were reported for the hybrid immobilization antigens in syngen 2 of $P$. aurelia (Finger et al., 1966). In Paramecium, only one of the possible 25 or so different molecular forms of the antigen was found in a single clone, although a random distribution of hybrid antigens was observed among the population of heterozygous clones. Nonrandomness in the aggregation of subunits has been observed during the synthesis of the hemoglobin molecule (Itano \& Robinson, 1960) and for some enzymes in vivo, such as the fish LDH's (Markert \& Faulhaber, 1965). Mammalian $\mathrm{LDH}$ appeared to associate randomly in vivo, although variations occurred in different cells, presumably as a result of differential synthesis of the component subunits by the LDH genes (Markert, 1963b; 1965). In the case of LDH-X (which contain "C" subunits; Blanco et al., 1964), Goldberg \& Hawtrey (1967) have obtained evidence which suggests that the pattern of synthesis of the LDH isozymes shifts during spermatogenesis. As the spermatogonium matures into the spermatocyte, the synthesis of the " $A$ " and " $B$ " subunits ceases at the time when the synthesis of the " $C$ " subunits begins.

The esterase-1 isozymes appear to be large molecules (Allen, unpublished), but little is known of their chemical composition. The isozymes occur in homozygotes and probably arise by epigenetic change. Parallel examples of this type of isozyme may be found for alcohol dehydrogenase in Drosophila (Grell et al., 1968), enolase in yeast (Pfleiderer, 1968) and alkaline phosphatase in Escherichia coli (Schlesinger \& Anderson, 1968). In all three cases, mutation affects all the isozymes simultaneously. The alkaline phosphatase isozymes show variations which are dependent upon culture conditions, a fact which was beautifully exploited in purifying two of the isozymes. Once purified, they were stable and could not be interconverted, but their kinetic properties were identical. However, when fingerprints were examined, a lysine-containing peptide was found in one isozyme which was missing from the other isozyme. Schlesinger \& Anderson (1968) have pointed out that the change in amino acid composition must be epigenetic, and they have speculated that it is brought about by some unknown cytoplasmic mechanism.

In the cases of both phosphatase-1 and esterase-1, there is evidence that the intracellular localization may be an important variable. The phosphatase-1 isozymes were localized to lysosomes (Allen et al., 1963b). It is possible that they are active only when lysosomes are present, although this point needs to be explored more fully. In a sense, this type of observation is reminiscent of the kind of interaction found by Munkres and Woodward (1966) for MDH and the mitochondrion. When the structural protein of the mitochondrion was 
defective, $\mathrm{MDH}$ presumably could not assume the proper stereospecific fit within the mitochondrial structure and was, therefore, inactive. Arguing by analogy, it is possible that the phosphatases require the presence of a lysosomal component in order that they may assume the configuration necessary for enzymatic activity. The intracellular localization of the esterase isozymes also varied, and it was suggested that each isozyme may be associated with a different cellular component (Allen, 1964b). Whether this does, in fact, occur, and why it should occur, are difficult questions to answer, especially since nothing is known of the in viw reactions catalyzed by these isozymes. To my knowledge, there are no close parallels to the situation observed with the esterase isozymes, although other isozymes, such as the LDH isozymes, do show differential intracellular localization. (See review by Vesell, 1965.) Such differential localization might be advantageous to a cell in that it could permit the use of the same enzyme in several metabolic pathways.

Regardless of the routes by which isozymes arise and are controlled in Tetrahymena and in other organisms, an isozymic system has one feature in common, and that is, it is composed of similar, yet diverse, molecules. Such a system has both redundancy and plasticity, and may permit experimentation with new molecular rearrangements while at the same time preserving the proven arrangements. It is of more than passing interest that redundancy creeps in so frequently, and at so many different levels of molecular organization. It is perhaps this characteristic that permits change and yet buffers a cell during changing environmental conditions.

\section{Summary}

Multiple forms of several different enzymes have been observed by gel electrophoresis in crude extracts of $T$. pyriformis, syngen 1. Examples of some of these are discussed. These multiple forms show variations which are of several types. Some variations are strain dependent. Structural genes have been identified for two sets of esterase isozymes and one set of acid phosphatase isozymes.

Variations also occur in heterozygotes which lead to cells with different stable phenotypes. This phenomenon was used to advantage in obtaining indirect structural information on the acid phosphatases in heterozygotes. Five phosphatase isozymes were observed, but not all were present in all cells. Seven cell types could be distinguished by their ability to synthesize one, three, or all five of the isozymes. These studies suggested that, if the phosphatase molecule is a tetramer, then, instead of random aggregation of the monomers, dimerization occurs as a first step. There appear to be three types of dimers, and these associate at random. Highly stable cell types synthesize only one of the three types of dimers.

Variations in the activity of isozymes occur under different growth conditions. For example, certain esterase isozymes, which appear in homozygotes and are nonrandomly distributed within the cell, varied in activity during the 
growth cycle. They could be converted into new isozymes by chemical treatment in vitro. One of the isozymes was localized to the microsomes and was enriched under logarithmic conditions of growth. It was speculated that the other isozymes may normally be elaborated from this isozyme, perhaps by conformational isomerism, or by some unknown epigenetic change, which has to do with their attachment to different intracellular structures.

\section{References}

ALLEN, S. L. 1960. Inherited variations in the esterases of Tetrahymena. Genetics 45: $1051-1070$.

ALLEN, S. L. 1961. Genetic control of the esterases in the protozoan Tetrahymena pyriformis. Ann. N. Y. Acad. Sci. 94: 753-773.

ALLEN, S. L. 1964a. Linkage studies in variety of 1 of Tetrahymena pyriformis: a first case of linkage in the ciliated protozoa. Genetics 49: 617-627.

ALLEN, S. L. 1964b. The esterase isozymes of Tetrahymena: Their distribution in isolated cellular components and their behavior during the growth cycle. J. Exp. Zool. 155: $349-370$.

ALLEN, S. L. 1965. Genetic control of enzymes in Tetrahymena. Brookhaven Sympos. Biol. 18: 27-52.

ALLEN, S. L. 1967a. Genomic exclusion: a rapid means for inducing homozygous diploid lines in Tetrahymena pyriformis, syngen 1. Science 155: 575-577.

ALLEN, S. L. 1967b. Cytogenetics of genomic exclusion in Tetrahymena. Genetics 55: 797-822.

ALLEN, S. L. \& D. L. NANNEY. 1958. An analysis of nuclear differentiation in the selfers of Tetrahymena. Am. Naturalist 92: 139-160.

ALLEN, S. L., J. M. ALLEN \& B. M. LICHT. 1965. Effects of Triton X-100 upon the activity of some electrophoretically separated acid phosphatases and esterases. J. Histochem. Cytochem. 13: 434-440.

ALLEN, S. L., S. K. FILE \& S. L. KOCH. 1967. Genomic exclusion in Tetrahymena. Genetics 55: 823-837.

ALLEN, S. L., M. S. MISCH \& B. M. MORRISON. 1963a. Genetic control of an acid phosphatase in Tetrahymena: formation of a hybrid enzyme. Genetics 48: 1635-1658.

ALLEN, S. L., M. S. MISCH \& B. M. MORRISON. 1963b. Variations in the electrophoretically separated acid phosphatases of Tetrahymena. J. Histochem. Cytochem. 11: 706-719.

ALPERS, D. A., E. STEERS, S. SHIFRIN \& G. M. TOMKINS. 1968. Isoenzymes of the lactose operon of Escherichia coli. Ann. N. Y. Acad. Sci. This monograph.

APPELLA, E. \& C. L. MARKERT. 1961. Dissociation of lactate dehydrogenase into subunits with guanidine-HCl. Biochem. Biophys. Res. Commun. 6: 171-176.

BLANCO, A., W. H. ZINKHAM \& L. KUPCHYK. 1964. Genetic control and ontogeny of lactate dehydrogenase in pigeon testes. J. Exp. Zool. 156: 137-152.

BLEYMAN, L. K., E. M. SIMON \& R. BROSI. 1966. Sequential nuclear differentiation in Tetrahymena. Genetics 54: 277-291.

DAVIS, B. J. 1964. Disc electrophoresis-II. Method and application to human serum proteins. Ann. N. Y. Acad. Sci. 121: 404-427.

DRYL, S. 1959. Antigenic transformation in Paramecium aurelia after homologous antiserum treatment during autogamy and conjugation. J. Protozool. 6 (Suppl.): 25.

EDSALL, J. T. 1968. Multiple molecular forms of carbonic anhydrase in erythrocytes. Ann. N. Y. Acad. Sci. This monograph.

FINGER, I., F. ONORATO, C. HELLER \& H. B. WILCOX, III. 1966. Biosynthesis and structure of Paramecium hybrid antigen. J. Molec. Biol. 17: 86-101.

GOLDBERG, E. \& C. HAWTREY. 1967. The ontogeny of sperm specific lactate dehydrogenase in mice. J. Exp. Zool. 164: 309-316.

GRELL, E. H., K. B. JACOBSON \& J. B. MURPHY. 1968. Alterations of genetic material for analysis of alcohol dehydrogenase isozymes in Drosophila melanogaster. Ann. N. Y. Acad. Sci. This monograph. 
ITANO, H. A. \& E. ROBINSON. 1960. Specific recombination of the subunits of human hemoglobin. Ann. N. Y. Acad. Sci. 88: 642-654.

KIRKMAN, H. N. \& J. E. HANNA. 1968. Isozymes of red cell human glucose-6-phosphate dehydrogenase. Ann. N. Y. Acad. Sci. This monograph.

KITTO, G. B., P. M. WASSERMAN \& N. O. KAPLAN. 1966. Enzymatically active conformers of mitochondrial malate dehydrogenase. Proc. Nat. Acad. Sci. U. S. A. 56: $578-585$.

MARKERT, C. L. 1963a. Lactate dehydrogenase isozymes: dissociation and recombination of subunits. Science 140: 1329-1330.

MARKERT, C. L. 1963b. Epigenetic control of specific protein synthesis in differentiating cells. In Twenty-first Sympos. Soc. for the Study of Develop. and Growth. M. Locke, Ed. : 65-84. Academic Press, Inc., New York, N.Y.

MARKERT, C. L. 1965. Developmental genetics. In The Harvey Lectures, Series 59: 187-218. Academic Press, Inc. New York, N.Y.

MARKERT, C. L. \& I. FAULHABER. 1965. Lactate dehydrogenase isozyme patterns of fish. J. Exp. Zool. 159: 319-332.

MULLER, M. \& I. TÖRÖ. 1962. Studies on feeding and digestion in protozoa. III. Acid phosphatase activity in food vacuoles of Paramecium multimicronucleatum. J. Protozool. 9: 98-102.

MUNKRES, K. D. \& D. O. WOODWARD. 1966. On the genetics of enzyme locational specificity. Proc. Nat. Acad. Sci. U. S. A. 55: 1217-1224.

NANNEY, D. L. 1963. Aspects of mutual exclusion in Tetrahymena. In Biological Organization: Cellular and Supracellular Levels. R. J. C. Harris, Ed. : 91-109. Academic Press, Inc. New York, N.Y.

NANNEY, D. L. 1964. Macronuclear differentiation and subnuclear assortment in ciliates. In Twenty-third Sympos. Soc. Study of Develop. and Growth. M. Locke, Ed. : 253-273. Academic Press, Inc. New York, N.Y.

NANNEY, D. L. \& J.M. DUBERT. 1960 . The genetics of the $\mathrm{H}$ serotype system in variety 1 of Tetrahymena pyriformis. Genetics 45: $1335-1349$.

NANNEY, D. L., M. J. NAGEL \& R. W. TOUCHBERRY. 1964. The timing of $\mathrm{H}$ antigenic differentiation in Tetrahymena. J. Exp. Zool. 155: 25-42.

PFLEIDERER, G. 1968. Biochemical, immunological, and genetic investigations of the multiple forms of yeast enolase. Ann. N. Y. Acad. Sci. This monograph.

PHILLIPS, R. B. 1967a. Inheritance of T serotypes in Tetrahymena. Genetics 56: 667-681.

PHILLIPS, R. B. 1967b. T serotype differentiation in Tetrahymena. Genetics 56: 683-692.

POLLOCK, M. R. 1968. The range and significance of variations amongst bacterial penicillinases. Ann. N. Y. Acad. Sci. This monograph.

SCHIMKE, R. T. \& L. GROSSBARD. 1968. Studies on isozymes of hexokinase in animal tissues. Ann. N. Y. Acad. Sci. This monograph.

SCHLESINGER, M. J. \& L. ANDERSON. 1968. Multiple molecular forms of the alkaline phosphatase of Escherichia coli. Ann. N. Y. Acad. Sci. This monograph.

SHAW, C. R. 1964. The use of genetic variation in the analysis of isozyme structure. Brookhaven Sympos. Biol. 17: 117-130.

SHAW, C. R. 1965. Electrophoretic variation in enzymes. Science 149: 936-943.

SONNEBORN, T. M. 1942. Inheritance in ciliate protozoa. Am. Naturalist 76: 46-62.

SUNDARAM, T. K. \& J. R. S. FINCHAM. 1964. A mutant enzyme in Neurospora crassa interconvertible between electrophoretically distinct active and inactive forms. J. Molec. Biol. 10: 423-437.

URSPRUNG, H. 1968. Multiple molecular forms of alcohol dehydrogenase in Drosophila melanogaster. Ann. N. Y. Acad. Sci. This monograph.

VESELL, E. S. 1965. Genetic control of isozyme patterns in human tissues. In Progress in Medical Genetics. H. G. Steinberg and H. G. Bearn, Eds. : 128-175. Grune and Stratton, New York, N.Y.

WELLNER, D. \& M. B. HAYES. 1968. Multiple molecular forms of L-amino acid oxidase. Ann. N. Y. Acad. Sci. This monograph. 